\title{
A note on small covers over cubes
}

\author{
Aslı Güçlükan İlhan (D) \\ Department of Mathematics, Faculty of Science, Dokuz Eylül University, Izmir, Turkey
}

\begin{abstract}
In this paper, we obtain a bijection between the weakly $\mathbb{Z}_{2}^{n}$-equivariant homeomorphism classes of small covers over an $n$-cube and the orbits of the action of $\mathbb{Z}_{2} \succ S_{n}$ on acyclic digraphs with $n$ vertices given by local complementation and reordering of vertices. We obtain a similar formula for the number of orientable small covers over an $n$-cube. We also count the $\mathbb{Z}_{2}^{n}$-equivariant homeomorphism classes of orientable small covers and estimate the ratio between this number and the number of $\mathbb{Z}_{2}^{n}$-equivariant homeomorphism classes of small covers over an $n$-cube.
\end{abstract}

Mathematics Subject Classification (2010). 37F20, 57S10

Keywords. small cover, weakly equivariant homeomorphism, acyclic digraph

\section{Introduction}

A small cover is an $n$-dimensional smooth closed manifold $M$ with a locally standard $\mathbb{Z}_{2}^{n}$-action whose orbit space is a simple convex polytope. One of the important problems of toric topology is to classify small covers over a given polytope (see $[2,3,5,8]$ ). For a given simple convex polytope $P$, let $\mathcal{F}(P)$ be the set of codimension one faces of $P$. For every small cover $M$ over $P$, there is an associated function $\lambda: \mathcal{F}(P) \rightarrow \mathbb{Z}_{2}^{n}$, called the characteristic function (see Section 2 for definition). Small covers $M_{1}$ and $M_{2}$ over $P$ are said to be Davis-Januskiewicz equivalent if there is a weakly $\mathbb{Z}_{2}^{n}$-equivariant homeomorphism between them which covers the identity on $P$. In [6], Davis and Januszkiewicz show that characteristic functions can be used to reconstruct small covers up to Davis-Januskiewicz equivalence. Moreover, they show that the characteristic functions that are in the same orbit of the standard free action of the general linear group give the same small cover up to Davis-Januskiewicz equivalence.

Another group that acts naturally on the set of characteristic functions on $P$ is the group of automorphisms of the face poset of $P$. In [10], Lü and Masuda show that there is a one-to-one correspondence between the orbit space of this action and the set of equivariant homeomorphism classes of small covers over $P$. Therefore the characteristic functions that are in the same double coset of these actions produce the same small cover up to weakly $\mathbb{Z}_{2}^{n}$-equivariant homeomorphism. Since the action of the automorphism group is not free, it is not easy to count the number of elements in these orbits.

The total space of iterated $\mathbb{R} P^{1}$ bundles starting with a point where each fibration is the projectivization of a Whitney sum of two real line bundles is called a real Bott manifold. Kamishima and Masuda [9] show that real Bott manifolds satisfy the cohomological rigidity

Email address: asli.ilhan@deu.edu.tr

Received: 10.10.2019; Accepted: 16.03.2020 
property, that is, two real Bott manifolds are diffeomorphic if and only if there is a graded ring ismorphism between their cohomology rings with $\mathbb{Z}_{2}$-coefficients. This also shows that their homeomorphism and diffeomorphism classes are the same. It is known that every real Bott manifold is a small cover over a cube and conversely, every small cover over a cube is homeomorphic to a real Bott manifold (see [11]). For this reason, small covers over $n$-cubes are studied intensively. In [3], Choi constructs a bijection from the set of Davis-Januszkiewicz equivalence classes of small covers over an $n$-cube to the set of acyclic digraphs with $n$ labeled nodes. Choi [3] also obtains a formula for the number of $\mathbb{Z}_{2}^{n}$ equivariant homeomorphism classes of small covers over an $n$-cube. In [5], Choi, Masuda and Oum introduce a notion of a Bott equivalence on acyclic digraphs and they show that two real Bott manifolds are diffeomorphic if and only if the associated digraphs are Bott equivalent. This also classifies the small covers over an $n$-cube up to homeomorphism. Using this correspondence and the list of non-isomorphic acyclic digraphs provided by B. D. McKay [14], they count the number of Bott manifolds up to dimension eight. Before that the number of Bott manifolds was known up to 5. Unfortunately, there is no known formula giving this number directly.

In this paper, we obtain a one-to-one correspondence between weakly $\mathbb{Z}_{2}^{n}$-equivariant homeomorphism classes of small covers over an $n$-cube and the orbit space of the action of $\mathbb{Z}_{2}$ 々 $S_{n}$ on acyclic digraphs with $n$ labeled vertices given by local complementation and reordering of vertices (see Theorem 3.4). The number of weakly $\mathbb{Z}_{2}^{n}$-equivariant homeomorphism classes of small covers over an $n$-cube gives an upper bound for the number of homeomorphism classes and hence the diffeomorphism classes of real Bott manifolds. Counting the size of the orbits of the action of $\mathbb{Z}_{2} \succ S_{n}$ on acyclic digraphs seems difficult but more computable than the number of Bott equivalence classes of digraphs. We also give a similar correspondece for the number of orientable small covers over an $n$-cube up to weakly $\mathbb{Z}_{2}^{n}$-equivariant homeomorphism.

In [4], Choi determines the number of Davis-Januskiewicz equivalence classes of orientable small covers over an $n$-cube and approximates the ratio $O_{n} / R_{n}$ where $O_{n}$ is the number of orientable small covers and $R_{n}$ is the number of small covers over an $n$-cube up to Davis-Januskiewicz equivalence. In Section 4, we obtain a recursive formula for the number of orientable small covers over an $n$-cube up to $\mathbb{Z}_{2}^{n}$-equivariant homeomorphism (see Theorem 4.2). We also show that the ratio between this number and the number of small covers up to $\mathbb{Z}_{2}^{n}$-equivariant homeomorphism is approximately $\frac{2.26}{2^{n}}$.

This paper is organized as follows. In Section 2, we give the necessary background on small covers. Section 3 is devoted the classification of small covers over an $n$-cube up to weakly $\mathbb{Z}_{2}^{n}$-equivariant homeomorphism. In Section 4 , we give a formula for the number of orientable small covers over an $n$-cube up to $\mathbb{Z}_{2}^{n}$-equivariant homeomorphism.

\section{Preliminaries}

Let $P$ be a simple convex polytope of dimension $n$. A small cover over $P$ is a smooth closed $n$-manifold endowed with a $\mathbb{Z}_{2}^{n}$-action that satisfies the following conditions:

i) The action is locally isomorphic to the standard action of $\mathbb{Z}_{2}^{n}$ on $\mathbb{R}^{n}$,

ii) The orbit space of the action is homeomorphic to $P$.

Small covers $M_{1}$ and $M_{2}$ over $P$ are said to be Davis-Januszkiewicz equivalent (DJequivalent) if there is a weakly $\mathbb{Z}_{2}^{n}$-equivariant homeomorphism $f: M_{1} \rightarrow M_{2}$ covering the identity on $P$.

Given a simple convex polytope $P$ of dimension $n$, let $\mathcal{F}(P)=\left\{F_{1}, \ldots, F_{m}\right\}$ be the set of facets of $P$. A function $\lambda: \mathcal{F}(P) \rightarrow \mathbb{Z}_{2}^{n}$ is said to satisfy the non-singularity condition if whenever the facets $F_{i_{1}}, \cdots, F_{i_{n}}$ meet at a vertex then $\left\{\lambda\left(F_{i_{1}}\right), \ldots, \lambda\left(F_{i_{n}}\right)\right\}$ is a basis for $\mathbb{Z}_{2}^{n}$. Such a function is called a characteristic function on $P$. We denote the set of all characteristic functions on $P$ by $\Lambda(P)$. Every point $p \in P$ is contained in the 
relative interior of a unique face, say $F(p)$. Let $G_{F(p)}$ be the subgroup of $\mathbb{Z}_{2}^{n}$ generated by $\lambda\left(F_{i_{1}}\right), \ldots, \lambda\left(F_{i_{k}}\right)$ where $F_{i_{1}}, \cdots, F_{i_{k}}$ are the set of all facets containing $F(p)$. Then the manifold $M(\lambda)=\left(P \times \mathbb{Z}_{2}^{n}\right) / \sim$ where the equivalence relation is defined by

$$
(p, g) \sim(q, h) \text { if } p=q \text { and } g^{-1} h \in G_{F(p)}
$$

is a small cover over $P$.

Theorem 2.1. [6] For every small cover $M$ over $P$, there is a characteristic function $\lambda$ with $\mathbb{Z}_{2}^{n}$-equivariant homeomorphism $M(\lambda) \rightarrow M$ covering the identity on $P$.

The general linear group $G L\left(n, \mathbb{Z}_{2}\right)$ acts freely on $\Lambda(P)$ by composition. The characteristic functions in the same orbit induce DJ-equivalent small covers. Therefore the DJ-equivalence classes of small covers over $P$ bijectively corresponds to the cosets $G L\left(n, \mathbb{Z}_{2}\right) \backslash \Lambda(P)$ by the above theorem. A standard way to count the number of orbits is to work with $(n \times m)$-matrices associated to characteristic functions. More precisely, for every $\lambda$, there is an associated matrix

$$
\Lambda=\left(\lambda\left(F_{1}\right), \cdots, \lambda\left(F_{m}\right)\right) .
$$

By reordering the facets and choosing a basis, we can choose a representative of the orbit of $\Lambda$ of the form $\left(I_{n} \mid \Lambda_{*}\right)$ where $\Lambda_{*}$ is an $(n \times(m-n))$-matrix. We call $\Lambda$ a characteristic matrix of $\lambda$ and $\Lambda_{*}$ the reduced submatrix of $\lambda$.

Another group that acts naturally on $\Lambda(P)$ is $\operatorname{Aut}(\mathcal{F}(P))$, the automorphism group of the face poset of $P$. The right action of $\operatorname{Aut}(\mathcal{F}(P))$ on $\Lambda(P)$ is also defined by composition. In [10], Lü and Masuda prove that the equivariant classes of small covers over $P$ are characterized by this action.

Theorem 2.2. [10] The set of $\mathbb{Z}_{2}^{n}$-equivariant homeomorphism classes of small covers over $P$ corresponds bijectively to the coset $\Lambda(P) / \operatorname{Aut}(\mathcal{F}(P))$.

By the above theorem the number of equivariant classes of small covers over $P$ is equal to the number of orbits of $\Lambda(P)$ under the action of Aut $(\mathcal{F}(P))$. Therefore Burnside's Lemma reduces the problem of counting the number of equivariant homeomorphism classes of small covers over $P$ to an enumeration of characteristic functions that are fixed by an element of $\operatorname{Aut}(\mathcal{F}(P))$.

One can combines above theorems to enumerate the number of weakly $\mathbb{Z}_{2}^{n}$-equivariant small covers over $P$.

Theorem 2.3. The set of weakly $\mathbb{Z}_{2}^{n}$-homeomorphism classes of small covers over $P$ corresponds bijectively the double cosets $G L\left(n, \mathbb{Z}_{2}\right) \backslash \Lambda(P) / \operatorname{Aut}(\mathcal{F}(P))$.

In [12], Nakayama and Nishimura give the following simple criterion for a small cover to be orientable.

Theorem 2.4. [12] For a basis $\left\{e_{1}, \cdots, e_{n}\right\}$ of $\mathbb{Z}_{2}^{n}$, let the homomorphism $\epsilon: \mathbb{Z}_{2}^{n} \rightarrow \mathbb{Z}_{2}$ be defined by $\epsilon\left(e_{i}\right)=1$ for $i=1, \cdots, n$. Then a small cover $M(\lambda)$ over a simple convex polytope $P$ of dimension $n$ is orientable if and only if there exists a basis $\left\{e_{1}, \cdots, e_{n}\right\}$ of $\mathbb{Z}_{2}^{n}$ such that the image of the composition $\epsilon \circ \lambda$ is $\{1\}$.

By the above theorem a small cover $\mathrm{M}$ with a characteristic matrix $\Lambda$ is orientable if and only if the sum of entries of the $j$-th column of $\Lambda$ is odd for $1 \leq j \leq m$. A characteristic function satifying the orientability condition given in the above theorem is called orientable. The action of $\operatorname{Aut}(\mathcal{F}(P))$ on the set of characteristic functions iduces an action on the orientable ones. Hence the number of equivariant and weakly equivariant small covers over $P^{n}$ are equal to the number of orbit spaces of the actions of $\operatorname{Aut}(\mathcal{F}(P))$ and $G L\left(n, \mathbb{Z}_{2}\right) \times \operatorname{Aut}(\mathcal{F}(P))$ on the set of orientable characteristic functions, respectively. 


\section{Weakly equivariant classification of small cover over an $n$-cube}

In this section, we classify the weakly $\mathbb{Z}_{2}^{n}$-equivariant classes of small covers over an $n$-cube in terms of acyclic digraphs. An $n$-dimensional cube $I^{n}$ has $2 n$ facets and for each facet there is only one facet that does not intersect it. Following Choi ([3]), we order the facets of $I^{n}$ so that $F_{i}$ and $F_{i+j}$ do not intersect for $1 \leq i \leq n$. In this case $\Lambda_{*}=\left(a_{i j}\right)$ is an $(n \times n)$-matrix and the non-singularity condition holds if and only if every principal minor of $\Lambda_{*}$ is 1 (see [3]). This means that there is a bijection between the set of DJ-equivalence classes of small covers over an $n$-cube and the set $M(n)$ of $(n \times n)$ matrices over $\mathbb{Z}_{2}$ all of whose principal minors are 1 . Here every diagonal of $\Lambda_{*}$ is a principal minor and hence $a_{i i}=1$ for $1 \leq i \leq n$. Moreover the $(2 \times 2)$-matrix $\left(\begin{array}{cc}a_{i i} & a_{i j} \\ a_{j i} & a_{j j}\end{array}\right)$ is a principal minor of $\Lambda_{*}$ and hence $a_{i j} a_{j i}=0$ for every $1 \leq i \neq j \leq n$. Therefore $\Lambda_{*}-I_{n}$ is equal to an adjacency matrix of some digraph. Choi [3] shows that the corresponding digraph is acyclic and the correspondence is a bijection.

Theorem 3.1 (Theorem 2.2, [3]). There is a bijection between the DJ-equivalence classes of small covers over $I^{n}$ and the set of acyclic digraphs with $n$ labeled vertices.

Recall that a digraph is a graph where the each edge has a direction. The edge from a vertex $v$ to a vertex $w$ is denoted by $[v, w]$. We denote the set of vertices of $G$ by $V(G)$ and the set of edges of $G$ by $E(G)$. If $[v, w] \in E(G)$ then we say that $v$ is an in-neighbor of $w$ and $w$ is an out-neighbor of $v$. For a vertex $v \in V(G)$, let $N_{G}^{+}(v)$ be the set of all out-neighbors of $v$ and $N_{G}^{-}(v)$ be the set of all in-neighbors of $v$. Given a directed graph $G$ with $V(G)=\left\{v_{1}, \cdots, v_{n}\right\}$, the adjacency matrix of $G$ is an $(n \times n)$-matrix whose $(i, j)$-entry is 1 if $[v(i), v(j)] \in E(G)$, and 0 , otherwise. A digraph is said to be acyclic if there is no cycle in which each edge is traversed in the same direction. Let $\mathcal{G}_{n}$ be the set of acyclic digraphs with $n$ labeled vertices and $R_{n}$ be the number of such digraphs.

Theorem 3.2. [13] The number of acyclic digraphs with $n$ labeled vertices is given by the following recursive formula:

$$
R_{n}=\sum_{k=1}^{n}(-1)^{k+1}\left(\begin{array}{l}
n \\
k
\end{array}\right) 2^{k(n-k)} R_{n-k} .
$$

By theorem 2.3, the number of weakly $\mathbb{Z}_{2}^{n}$-equivariant homeomorphism classes of small covers over $I^{n}$ is equal to $\left|M(n) / \operatorname{Aut}\left(\mathcal{F}\left(I^{n}\right)\right)\right|$. Here the action of $\operatorname{Aut}\left(\mathcal{F}\left(I^{n}\right)\right)$ on $M(n)$ is induced from its action on the set of characteristic functions on $I^{n}$. It is well-known that $\operatorname{Aut}\left(\mathcal{F}\left(I^{n}\right)\right)$ is the wreath product $\mathbb{Z}_{2} \prec S_{n}$ of $\mathbb{Z}_{2}$ by $S_{n}$. Let us denote the involution that interchanges $F_{i}$ and $F_{n+i}$ and fixes other faces by $\chi_{i}$. Then every element of this group can be written in the form $\mu \cdot \chi_{1}^{e_{1}} \cdots \chi_{n}^{e_{n}}$ where $\mu \in S_{n}$ and $e_{i} \in \mathbb{Z}_{2}$. Here a permutation $\mu \in S_{n}$ sends $F_{j}$ to $F_{\mu(j)}$ and $F_{n+j}$ to $F_{n+\mu(j)}$ for $1 \leq j \leq n$. Let $\Lambda_{*}$ be the $(n \times n)$ reduced submatrix of a given characteristic function $\lambda$. Then for any $\mu \in S_{n}$ we have

$$
\left(I_{n} \mid \Lambda_{*}\right) \cdot \mu=\left(I_{n} \mid P(\mu)^{-1} \Lambda_{*} P(\mu)\right) .
$$

Under the bijection given by Theorem 3.1, this action corresponds to the standard action of $S_{n}$ on the set of acyclic digraphs by permuting the vertices. Using this, Choi (Theorem $4.1,[3])$ shows that $\left|M(n) / \operatorname{Aut}\left(\mathcal{F}\left(I^{n}\right)\right)\right|$ is less than equal to the number of unlabeled acyclic digraphs with $n$-vertices. In this section, we give a complete classification of small covers over an $n$-cube up to weakly $\mathbb{Z}_{2}^{n}$-equivariant homeomorphism. For this we need the following definition.

Definition 3.3. ([7]) Let $G$ be a digraph and $v$ be a vertex in $G$. The local complementation of $G$ at $v$ is the acyclic digraph $G * v$ with $V(G * v)=V(G)$ and $E(G * v)$ is the symmetric difference of sets $E(G)$ and $\left\{[u, w] \mid(u, w) \in N_{G}^{-}(v) \times N_{G}^{+}(v)\right\}$. 
In other words, the local complementation of $G$ at $v$ is obtained by removing $[u, w]$ from $G$ if it is an edge of $G$ or adding [u,w], otherwise for every in-neighbor $u$ and out-neighbor $w$ of $v$.
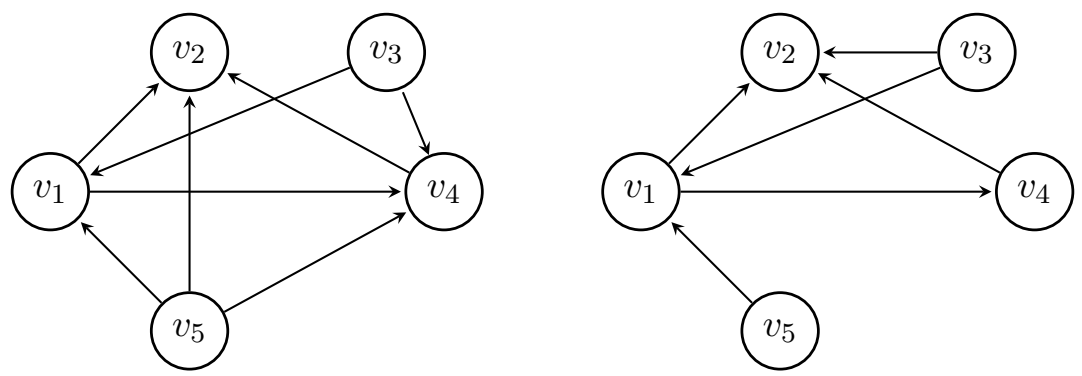

Figure 1. Local complementation at $v_{1}$.

Theorem 3.4. There is a bijection between the set of weakly $\mathbb{Z}_{2}^{n}$-equivariant homeomorphism classes of small covers over $I^{n}$ and the orbit space of the action of $\mathbb{Z}_{2}$ ? $S_{n}$ on $\mathcal{G}_{n}$ where $\mu \in S_{n}$ acts by permuting the vertices and $\chi_{i}$ acts as local complementation at $v_{i}$.

Proof. Let $\lambda$ be a characteristic function on $I^{n}$ with a reduced submatrix $\Lambda_{*}=\left(a_{p q}\right)$. This means that we can represent the equivalence class of $\lambda$ in $G L\left(n, \mathbb{Z}_{2}\right) \backslash \Lambda\left(I^{n}\right)$ by $\left(I_{n} \mid \Lambda_{*}\right)$. Since $\chi_{i}$ acts on faces by interchanging $F_{i}$ and $F_{n+i}$, we have

$$
\left(I_{n} \mid \Lambda_{*}\right) \cdot \chi_{i}=\left(P_{i}\left(\Lambda_{*}\right) \mid Q_{i}\left(\Lambda_{*}\right)\right),
$$

where $P_{i}\left(\Lambda_{*}\right)$ is the $(n \times n)$-matrix obtained by replacing the $i$-th column of the identity with the $i$-the column of $\Lambda_{*}$ and $Q_{i}\left(\Lambda_{*}\right)$ is the $(n \times n)$-matrix obtained by replacing the $i$-th column of $\Lambda_{*}$ with the $i$-the column of the identity. Since the diagonals of $\Lambda_{*}$ are all 1 and we are working with matrices over $\mathbb{Z}_{2}$, the invertible matrix $P_{i}\left(\Lambda_{*}\right)$ is involutary, i.e, $P_{i}\left(\Lambda_{*}\right)^{-1}=P_{i}\left(\Lambda_{*}\right)$. Therefore we have $\left(I_{n} \mid \Lambda_{*}\right) \cdot \chi_{i}=P_{i}\left(\Lambda_{*}\right) \cdot\left(I_{n} \mid P_{i}\left(\Lambda_{*}\right) \cdot Q_{i}\left(\Lambda_{*}\right)\right)$. Clearly $(p, q)$-entry of $P_{i}\left(\Lambda_{*}\right) \cdot Q_{i}\left(\Lambda_{*}\right)$ is $a_{p q}$ if $i \in\{p, q\}$ and $a_{p i} a_{i q}+a_{p q}$, otherwise. Therefore $\chi_{i}$ sends orbit of $\lambda$ with $\Lambda_{*}=\left(a_{p q}\right)$ in $G L\left(n, \mathbb{Z}_{2}\right) \backslash \Lambda\left(I^{n}\right)$ to a orbit represented by $\left(I_{n} \mid\left(b_{p q}\right)\right)$ where

$$
b_{p q}= \begin{cases}a_{p q} & \text { if } i \in\{p, q\} \\ a_{p i} a_{i q}+a_{p q} & \text { otherwise. }\end{cases}
$$

Let $G$ be the acyclic digraphs with labeled vertices $\left\{v_{1}, \cdots, v_{n}\right\}$ and the adjacency matrix $\Lambda_{*}-I_{n}$. Then the induced action of $\chi_{i}$ sends $G$ to a digraph $G^{\prime}$ with the adjaceny matrix $\left(b_{p q}\right)-I_{n}$. Here, $b_{p q}$ is different than $a_{p q}$ if and only if $v_{p}$ is an in-neighbor and $v_{q}$ is an out-neighbor of $v_{i}$ in $G$. In this case, $\left[v_{p}, v_{q}\right]$ is an edge of $G^{\prime}$ if and only if $\left[v_{p}, v_{q}\right]$ is not an edge of $G$. This means that $G^{\prime}$ is obtained from $G$ by removing $\left[v_{p}, v_{q}\right]$ from $G$ if it is an edge of $G$ or adding $\left[v_{p}, v_{q}\right]$, otherwise whenever $v_{p}$ is an in-neighbor and $v_{q}$ is an out-neighbor of $v_{i}$ in $G$. Therefore $\chi_{i}$ sends $G$ to the local complementation of $G$ at $v_{i}$.

Since the number of weakly $\mathbb{Z}_{2}^{n}$-equivariant homeomorphism classes gives an upper bound for the number of homeomorphism classes, as an immediate consequence of the above theorem we obtain the following corollary.

Corollary 3.5. The number of small covers of small covers over $I^{n}$ up to homeomorphism is less than or equal to the size of the orbit space of the action of $\mathbb{Z}_{2} 2 S_{n}$ on $\mathcal{G}_{n}$ where $\mu \in S_{n}$ acts by permuting the vertices and $\chi_{i}$ acts as local complementation at $v_{i}$.

Remark 3.6. The local complementation of a digraph at a vertex also appears in the classification of small covers over cubes up to homeomorphisms (see [5]). In [5], Choi, Masuda and Oum show that level types, rank, odd height and sibling classes of associated 
acyclic digraphs are invariant under homeomorphism classes of small covers over an $n$-cube. Therefore these are also invariants of weakly equivariant homeomorphism classses. In [1], Bouchet shows that local complementation also preserves cut-rank functions. However, it is not an invariant of homeorphism classes of small covers over an $n$-cube (see [5]). It is easy to see that cut-rank is also invariant under the reordering of vertices and hence it is an invariant of weakly $\mathbb{Z} / 2$-equivariant homeomorphism classes of small covers.

By counting the orbits of the action of $\mathbb{Z}_{2} \succ S_{n}$ on the set of acyclic digraphs with $n$ labeled vertices, one can obtain an upper bound for the number of homeomorphism classes of small covers, which is known up to $n=8$ ([5]). By Burnside's Lemma, this number can be found counting the number of elements that are fixed by elements of $\mathbb{Z}_{2}<S_{n}$. Clearly when $n=2$, the number of orbits of the $\mathbb{Z}_{2} \prec \mathbb{Z}_{2}$-action is 2 .

Example 3.7. As listed in Figure 2, there are 5 different weakly $\mathbb{Z}_{2}^{n}$-equivariant homeomorphism classes of small cover over $I^{3}$. However, the number of homeomorphism classes of small covers over $I^{3}$ is 4 . In this case, Class 2 and Class 4 represent the same homeomorphism class (see [5]).

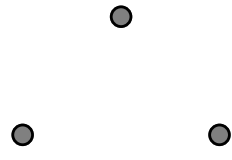

Class 1

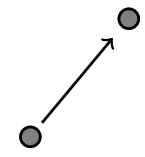

Class 2

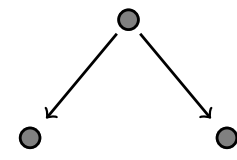

Class 3

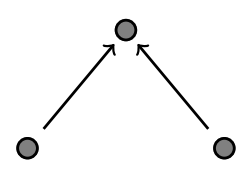

Class 4

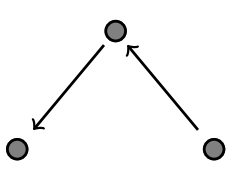

Class 5

Figure 2. Representatives of orbits of $\mathbb{Z}_{2} 2 S_{3}$-action on the set of acyclic digraphs with 3 labeled vertices.

With a custom SAGE implementation, we calculate the size of fixed points of elements of the automorphism group of the face poset of $I^{4}$ and obtain that the number of weakly equivariant homeomorphism classes of small covers over $I^{4}$ is 19 . In this case the number of DJ-equivalence classes, equivariant homeomorphism and homeomorphism classes of small covers over $I^{4}$ are 543, 259, and 12, respectively. In the following example, we give the size of these fixed points.

Example 3.8. Recall that an arbitrary element $g$ of $\operatorname{Aut}\left(\mathcal{F}\left(I^{4}\right)\right)$ is of the form $\mu \cdot \chi_{1}^{e_{1}} \cdots \chi_{4}^{e_{4}}$ where $\mu \in S_{4}, \chi_{i} \in \mathbb{Z} / 2$ and $e_{i} \in\{0,1\}$. If $\mu$ is the identity, then we have

$$
\left|\left(G L\left(n, \mathbb{Z}_{2}\right) \backslash \Lambda\left(I^{4}\right)\right)^{g}\right|= \begin{cases}543 & \text { if } g=\mathrm{id} \\ 375 & \text { if } g=\chi_{i}, 1 \leq i \leq 4, \\ 247 & \text { if } g=\chi_{i} \chi_{j}, 1 \leq<j \leq 4, \\ 159 & \text { if } g=\chi_{i} \chi_{j} \chi_{k}, 1<i<j<k \leq 4, \\ 111 & \text { if } g=\chi_{1} \chi_{2} \chi_{3} \chi_{4} .\end{cases}
$$

When the cycle type of $\mu$ is $(2,2),(3,1)$ or $(4)$, the number of fixed points of $g$ does not depend on the choice of $\left\{e_{1}, e_{2}, e_{3}, e_{4}\right\}$ and is 7,3 , and 1 , respectively. When the cycle 
type of $\mu$ is $(2,1,1)$, say $\mu=(a, b)$, the number of fixed points of $g$ is given as follows:

$$
\left|\left(G L\left(n, \mathbb{Z}_{2}\right) \backslash \Lambda\left(I^{4}\right)\right)^{g}\right|= \begin{cases}21 & \text { if } g=(a, b) \cdot \chi_{i} \text { with } i \in\{a, b\} \\ 21 & \text { if } g=(a, b) \cdot \chi_{i} \chi_{j} \text { with }|\{a, b\} \cap\{i, j\}|=1, \\ 21 & \text { if } g=(a, b) \cdot \chi_{i} \chi_{j} \chi_{k} \text { with }|\{a, b\} \cap\{i, j, k\}|=1, \\ 25 & \text { otherwise. }\end{cases}
$$

Therefore the number of weakly equivariant small covers over $I^{4}$ is

$$
\frac{543+375 \cdot 4+247 \cdot 6+159 \cdot 4+111+7 \cdot 48+3 \cdot 128+1 \cdot 96+21 \cdot 48+25 \cdot 48}{24 \cdot 16}=19
$$

by Burnside's Lemma.

Remark 3.9. The size of fixed points of $g=\chi_{i}$ can be counted easily even in the general case. Indeed, an acyclic digraph $G$ with labeled vertex set $\left\{v_{1}, \cdots, v_{n}\right\}$ is fixed by $\chi_{i}$ if and only if either there is no edge directed from $v_{i}$ (i.e, $v_{i}$ is a source of $G$ ) or there is no edge directed to $v_{i}$ (i.e, $v_{i}$ is a sink of $G$ ). By inclusion-exclusion principle, the number of such digraphs is

$$
2^{n-1} R_{n-1}+2^{n-1} R_{n-1}-R_{n-1}=\left(2^{n}-1\right) R_{n-1} .
$$

Therefore when $n=4,\left|\left(G L\left(n, \mathbb{Z}_{2}\right) \backslash \Lambda\left(I^{4}\right)\right)^{\chi_{i}}\right|=375$. One can compute the fixed points of $\chi_{i} \chi_{j}$ and $\chi_{i} \chi_{j} \chi_{k}$, similarly but it is not easy to find a formula giving the size of the fixed point set of $\chi_{i_{1}} \cdots \chi_{i_{k}}$ in general. It is an open and important problem of graph theory since it gives the number of equivalence classes of digraphs under local complementations by Burnside's Lemma.

\section{Orientable small covers over an $n$-cube}

Let $O(n)$ be the number of orientable small covers over an $n$-cube up to DJ-equivalence. In [4], Choi shows that $O(n)$ is equal to the number of acyclic digraphs all of whose vertices have even out-degree and hence obtains the following formula

$$
O(n)=\sum_{k=1}^{n}(-1)^{k+1}\left(\begin{array}{l}
n \\
k
\end{array}\right) 2^{(k-1)(n-k)} R_{n-k}
$$

and shows that the ratio $O(n) / R(n)$ is approximately $\frac{1.262}{2^{n}}$.

Theorem 4.1. (Theorem 3.3, [3]) Let ER(n) denote the number of $\mathbb{Z}_{2}^{n}$-equivariant homeomorphisms classes of small covers over an n-cube. Then

$$
E R(n)=\frac{\sum_{k=0}^{n}\left(\begin{array}{c}
n \\
k
\end{array}\right) 2^{k(n-i)} R_{n-k}}{2^{n} n !} \cdot \prod_{i=0}^{n-1}\left(2^{n}-2^{i}\right) .
$$

We obtain a similar formula for the number of orientable small covers over an $n$-cube. Although the proof is similar to that of the above theorem, we provide it for completeness.

Theorem 4.2. Let $E Q(n)$ denote the number of orientable small covers over an n-cube up to $\mathbb{Z}_{2}^{n}$-equivariant homeomorphisms. Then

$$
E Q(n)=\frac{\sum_{k=0}^{n}\left((-1)^{k+1}+1\right)\left(\begin{array}{l}
n \\
k
\end{array}\right) 2^{(k-1)(n-k)} R_{n-k}}{2^{n} n !} \cdot \prod_{i=0}^{n-1}\left(2^{n}-2^{i}\right) .
$$

Proof. Recall that an element $g$ of an automorphism group of the face poset of an $n$-cube is of the form $g=\mu \cdot \chi_{1}^{e_{1}} \cdots \chi_{n}^{e_{n}}$ where $\mu \in S_{n}$, the $\chi_{i}$ are reflections, and $e_{i} \in\{0,1\}$. If $\mu \neq 1$ then for any characteristic function $\lambda$, there exist $i, j$ with $n \nmid i-j$ such that $(\lambda \cdot g)\left(F_{i}\right)=(\lambda \cdot g)\left(F_{j}\right)$. This contradicts the non-singularity condition and hence the set of fix points of $g$ is empty. If $g=1$ then the number of fixed points of $g$ is the number of orientable small covers, which is given by formula 4.1. Now let, let $g=\chi_{1} \cdots \chi_{k}$ for some 
$k \geq 1$. Let $\lambda$ be an orientable characteristic function fixed by $g$ whose associated matrix is $\Lambda=(A \mid B)$. Since $g$ fixes $\lambda$, the first $k$ columns of $A$ and $B$ are the same. Therefore the reduced submatrix of $\lambda$ is of the form

$$
\Lambda_{*}=\left(\begin{array}{cc}
I_{k} & S \\
0 & T
\end{array}\right),
$$

where $I_{k}$ is the identity matrix of size $k, S$ is a $(k \times(n-k))$-matrix and $T$ is a square matrix of size $(n-k)$. Here $\Lambda_{*}$ is in $M(n)$ if and only if $T$ is in $M(n-k)$. By Theorem 2.4, $\lambda$ is orientable if and only if the sum of entries of $j$-th columns of $S$ and $T$ is odd for $1 \leq j \leq n-k$. By controlling only one row of $S$, we can ensure that $\Lambda_{*}$ satisfy this condition. Therefore the number of fixed point of $g$ is $\left|G L\left(n, \mathbb{Z}_{2}^{n}\right)\right| \times 2^{(k-1)(n-k)} R_{n-k}$ for $k \geq 1$. This number is independent of choices of reflections. Since the size of $G L\left(n, \mathbb{Z}_{2}^{n}\right)$ is $\prod_{i=0}^{n-1}\left(2^{n}-2^{i}\right)$, we have the required formula by Burnside's Lemma.

\begin{tabular}{|c|ccccccc}
\hline$n$ & 1 & 2 & 3 & 4 & 5 & 6 & $\cdots$ \\
\hline$E R(n)$ & 1 & 6 & 259 & 87360 & 236240088 & 5143046823936 & $\cdots$ \\
\hline$E Q(n)$ & 1 & 3 & 70 & 12180 & 16644768 & 181797614208 & $\cdots$ \\
\hline \multicolumn{7}{c}{ Table 1. Some values of $E R(n)$ and $E Q(n)$}
\end{tabular}

Now we estimate the ratio $E Q(n) / E R(n)$. Note that $E Q(n) / E R(n)=K_{n} / L_{n}$ where

$$
\begin{aligned}
K_{n} & =\sum_{k=0}^{n}\left((-1)^{k+1}+1\right)\left(\begin{array}{l}
n \\
k
\end{array}\right) 2^{(k-1)(n-k)} R_{n-k}, \\
L_{n} & =\sum_{k=0}^{n}\left(\begin{array}{l}
n \\
k
\end{array}\right) 2^{k(n-i)} R_{n-k} .
\end{aligned}
$$

Given a sequence $\left\{A_{n}\right\}$, let $A(x)$ be the chromatic generating function of $A_{n}$ :

$$
A(x)=\sum_{n=0}^{\infty} A_{n} \frac{x^{n}}{n ! 2^{\left(\begin{array}{c}
n \\
2
\end{array}\right)}} .
$$

Then $A(x)=B(x) C(x)$, where $A(x), B(x)$ and $C(x)$ are the chromatic generating functions of the sequences $\left\{A_{n}\right\},\left\{B_{n}\right\}$ and $\left\{C_{n}\right\}$, respectively, if and only if $A_{n}=$ $\sum_{k=0}^{n} B_{k} C_{n-k}\left(\begin{array}{l}n \\ k\end{array}\right) 2^{k(n-k)}$.

Lemma 4.3. Let $F(x)$ be the chromatic generating function of the constant sequence $F_{n}=1$. Then we have

$$
K(x)=\frac{F(x)-F(-x)}{F\left(-\frac{x}{2}\right)} \text { and } L(x)=\frac{F(x)}{F(-x)} .
$$

Proof. By Theorem 3.2, we have $F(-x) R(x)=1$, where $R(x)$ is the chromatic generating function of $R_{n}$. Therefore the second equality easily follows from Thereom 4.1. For the first one, write $K_{n}=K_{n}^{1}+K_{n}^{2}$ where

$$
\begin{aligned}
K_{n}^{1} & =\sum_{k=1}^{n}(-1)^{k+1}\left(\begin{array}{l}
n \\
k
\end{array}\right) 2_{n-k}^{(k-1)(n-k)} \\
K_{n}^{2} & =\sum_{k=1}^{n}\left(\begin{array}{l}
n \\
k
\end{array}\right) 2^{(k-1)(n-k)} R_{n-k} .
\end{aligned}
$$


Since $K^{1}(x)=R\left(\frac{x}{2}\right)\left(R\left(\frac{x}{2}\right)-F(-x)\right)$ and $K^{2}(x)=R\left(\frac{x}{2}\right)\left(F(x)-R\left(\frac{x}{2}\right)\right)$, we have

$$
K(x)=R\left(\frac{x}{2}\right)(F(x)-F(-x))=\frac{F(x)-F(-x)}{F\left(-\frac{x}{2}\right)} .
$$

Since $F(x)$ has an isolated zero at $\alpha=-1.488$ (see [4]), $L(x)$ also has an isolated zero at $\alpha$. Since $F^{\prime}(x)=F\left(\frac{x}{2}\right)$, we have

$$
L(x) \sim \frac{F^{\prime}(\alpha)}{F(-\alpha)}(x-\alpha)=\frac{F\left(\frac{\alpha}{2}\right)}{F(-\alpha)}(x-\alpha) .
$$

Therefore we have the formula

$$
L(x) \sim \frac{1}{L(-x)}=\frac{F(-\alpha)}{F\left(\frac{\alpha}{2}\right)(-x-\alpha)}=\frac{F(-\alpha)}{-\alpha F\left(\frac{\alpha}{2}\right)} \sum_{n=0}^{\infty}\left(-\frac{x}{\alpha}\right)^{n}
$$

and hence $L_{n} \sim A \cdot 2^{\left(\begin{array}{c}n \\ 2\end{array}\right)} n !\left(\frac{-1}{\alpha}\right)^{n}$ where $A=5.42$.

To estimate the behavior of $K_{n}$, let

$$
b(x)=\frac{F\left(\frac{x}{2}\right)}{F(-x)-F(x)} .
$$

It has an isolated zero at $2 \alpha$ and hence

$$
b(x) \sim \frac{F^{\prime}(\alpha)}{2(F(-2 \alpha)-F(2 \alpha))}(x-2 \alpha)=\frac{F\left(\frac{\alpha}{2}\right)}{2(F(-2 \alpha)-F(2 \alpha))}(x-2 \alpha) .
$$

Therefore, we have

$$
K(x)=\frac{1}{b(-x)} \sim \frac{2(F(-2 \alpha)-F(2 \alpha))}{F\left(\frac{\alpha}{2}\right)(-x-2 \alpha)}=\frac{F(-2 \alpha)-F(2 \alpha)}{-\alpha F\left(\frac{\alpha}{2}\right)} \sum_{n=0}^{\infty}\left(-\frac{x}{2 \alpha}\right)^{n} .
$$

It estimates $K_{n}$ as $B \cdot 2^{\left(\begin{array}{l}n \\ 2\end{array}\right)} n !\left(\frac{-1}{2 \alpha}\right)^{n}$ where $B=12.28$.

Corollary 4.4. $E Q(n) / E R(n) \sim \frac{2.26}{2^{n}}$.

As an immediate consequence of Theorem 3.4, we have the following classification for the weakly $\mathbb{Z}_{2}^{n}$-equivariant homeomorphism classes of orientable small covers over an $n$-cube.

Theorem 4.5. There is a bijection between the set of weakly $\mathbb{Z}_{2}^{n}$-equivariant homeomorphism classes of small covers over $I^{n}$ and the orbit space of the action of $\mathbb{Z}_{2} 2 S_{n}$ on the set of acyclic digraphs all of whose vertices have even out-degree by permuting the vertices and local complementations.

By above theorem, the number of orientable small covers over $I^{3}$ is 2 up to weakly $\mathbb{Z}_{2}^{n}$-equivariant homeomorphisms. They are represented by Class 1 and Class 3 of Figure 2.

Acknowledgment. We thank to referee for careful reading of the paper and useful comments. This work is supported by The Scientific and Technological Research Council of Turkey (Grant No: TBAG/118F310).

\section{References}

[1] A. Bouchet, Digraph decompositions and Eulerian systems, SIAM J. Algebraic Discrete Methods, 8, (3), 323-337, 1987.

[2] M. Cai, X. Chen and Z. Lü, Small covers over prisms, Topol. Appl. 154, 2228-2234, 2007. 
[3] S. Choi, The number of small covers over cubes, Algebr. Geom. Topol. 8, 2391-2399, 2008.

[4] S. Choi, The number of orientable small covers over cubes, Proc. Japan Acad. Ser. A, 86, 97-100, 2010.

[5] S. Choi, M. Masuda and S. Oum, Classification of real Bott manifolds and acyclic digraphs, Trans. Amer. Math. Soc. 369, 2987-3011, 2017.

[6] M.W. Davis and T. Januszkiewicz, Convex polytopes, Coxeter orbifolds and torus action, Duke Math. J. 62, 417-451, 1971.

[7] D.G. Fon-Der-Flaass, Local complementations of simple and directed graphs, in: Discrete Analysis and Operations Research, 1, 15-34, 1996.

[8] A. Garrison and R. Scott, Small covers over the dodecahedron and the 120-cell, Proc. Amer. Math. Soc. 131, 963-971, 2003.

[9] Y. Kamishima and M. Masuda, Cohomological rigidity of real Bott manifolds, Algebr. Geom. Topol. 9, 2479-2502, 2009.

[10] Z. Lü and M. Masuda, Equivariant classification of 2-torus manifolds, Colloq. Math. 115, 171-188, 2009.

[11] M. Masuda, T.E. Panov, Semi-free circle actions, Bott towers, and quasitoric manifolds, Mat. Sb. 199, 95-122. 2008.

[12] H. Nakayama and Y. Nishimura, The orientability of small covers and coloring simple polytopes, Osaka J. Math. 42, 243-256, 2005.

[13] V.I. Rodinov, On the number of labeled acyclic digraphs, Discrete Math. 105, 319-321, 1992.

[14] http://users.cecs.anu.edu.au/ bdm/data/digraphs.html. 